\title{
Violencia simbólica en las actividades laborales de las docentes en la Universidad Mayor de San Andrés La Paz - Bolivia
}

\author{
Symbolic violence in the work environment of teachers at Universidad Mayor \\ de San Andrés (University of San Andres), La Paz - Bolivia
}

ISSN 2071-8748

E-ISSN 2218-3345

(c) $(9)$

URI: http://hdl.handle.net/11298/881

DOI: http://dx.doi.org/10.5377/entorno.v0i66.6728
Valentina Rosario Alarcón-Velasco' alarconvalentina@yahoo.com

"Llamo desconocimiento al hecho de reconocer
una violencia que se ejerce precisamente
en la medida en que se le desconozca como violencia."
Pierre Bourdieu
Recibido: $15 / 08 / 18$
Aprobado: $12 / 12 / 18$

\section{Resumen}

En el presente trabajo investigativo se identificó y analizó las formas y expresiones de la violencia simbólica, bajo el enfoque teóricoy conceptual del sociólogo Pierre Bourdieu, las cuales suelen estar encubiertas en la sociedad. Éstas son ejercidas sobre mujeres que son docentes y autoridades universitarias que ocuparon u ocupan en la actualidad cargos jerárquicos en la Universidad Mayor de San Andrés (UMSA) en La Paz, Bolivia. Se constató la formación social de un menosprecio, discriminación y segregación contra la academia constituida por mujeres, la misma que influye en la configuración de la

\section{Abstract}

In the current research different forms and expressions of symbolic violence, which are usually hidden in society, were identified and analyzed under the theoretical and conceptual approach of the sociologist Pierre Bourdieu. These are exercised on women who are teachers and university authorities who currently occupy or occupied hierarchical positions at Universidad Mayor de San Andrés (UMSA) La Paz, Bolivia. The social formation of a contempt, discrimination and segregation against the academy constituted by women was confirmed and this influences the configuration of the political structure

1 Profesora de la Universidad Mayor de San Andrés de La Paz, Bolivia. Miembro de la Red de Género de Iberoamérica. 
estructura política de la citada universidad. Se encontró una reproducción social de las estructuras patriarcales que socavan las relaciones sociales e institucionales en la universidad. Para poder llegar a estos resultados, se utilizó un enfoque metodológico mixto, o lo que se ha denominado como metodología cuantitativa-cualitativa, concentrándose en lograr una mayor comprensión y explicación del estudio realizado. Con la metodología cuantitativa se pudo demostrar las diferencias numéricas de género con datos construidos con el apoyo del Departamento de Sistemas de Información Estadística (DSIE) de la UMSA y de ese modo se profundizó gracias a la aplicación de herramientas y técnicas de investigación de la metodología cualitativa, específicamente, se realizó una entrevista semi-estructurada a profundidad a 13 docente mujeres de todas las facultades de la universidad, se valoró la apertura de las entrevistadas para abordar un tema delicado y personal como el presentado en este trabajo investigativo.

\section{Palabras clave}

Estereotipo (psicología); Sociología de la educación; Violencia; Ambiente de trabajo; Estructuras patriarcales. of the aforementioned university. A social reproduction of the patriarchal structures that undermine social and institutional relations in the University was found. In order to reach these results, an integrated methodological approach, called quantitative-qualitative methodology was used, focusing mainly on achieving a greater understanding and explanation of the study conducted. With the quantitative methodology, it was possible to demonstrate the numerical differences of gender, based on the data obtained with the support of the Department of statistical information systems of the UMSA; thus the study deepened thanks to the application of tools and techniques of such methodology.

Specifically, a semi-structured in-depth interview was conducted with 13 female professors from all University faculties.; the openness of the interviewees to address such a delicate and personal topic as that presented in this research work was appreciated.

\section{Keywords}

Stereotype (psychology), Sociology of education, Violence, Work environment, Patriarchal structures.

\section{Introducción}

En Bolivia, el 9 de marzo de 2013 se decretó la ley No. 348 denominada "Ley Integral para Garantizar a las Mujeres una Vida Libre de Violencia", que indica 16 tipos de violencia contra la mujer, dentro de ella se tipifica a la denominada violencia simbólica. Sin embargo, hasta la fecha no se conoce de ningún caso ni estudio sobre el tema.

No obstante, los casos y estudios, como, por ejemplo, sobre violencia física y psicológica son más frecuentes, esto significa que a diferencia de la violencia simbólica se tiene cierta conceptualización, tanto jurídica como social, de que la violencia puede reconocerse en principio como física, pero también como psicológica. Por consiguiente, es posible encontrar una serie de castigos con base en la legislación mencionada, en la que se entiende a los hombres como principales sujetos de la agresión; y da la impresión de que el tema de la violencia es solamente causa de la mentalidad machista imperante en la sociedad; y pareciera expresar que la solución a este problema depende únicamente del cambio de actitud de estos. Se obvia, de hecho, que el tema de la violencia de género es histórico social enmarcado en las relaciones entre hombres y mujeres; en la construcción de sus relaciones en cuanto reproducción de la sociedad. 
La universidad es un campo de reproducción de relaciones sociales, en cuya estructura existe una división social del trabajo condicionado por el género, cuestión que se agrava a medida en que se va hurgando la dinámica de la propia universidad. En este sentido, la presente investigación está enmarcada en la Universidad Mayor de San Andrés (UMSA), creada por decreto supremo del 25 de octubre de 1830, a solo 5 años de la fundación de Bolivia; es catalogada como la principal casa de estudios superiores en Bolivia. Su régimen autonómico le permite tomar posición en el contexto nacional de una manera consciente, como ha ocurrido en las últimas décadas.

El enfoque de la investigación se basó en la teoría de la práctica del sociólogo Pierre Bourdieu, principalmente sus reflexiones y aportes sobre la violencia simbólica. Sin embargo, deben tomarse en cuenta otros dos conceptos que complementan su perspéctiva: habitus y dominación masculina.

\section{El habitus lo define Bourdieu como un}

sistema de disposiciones duraderas y transferibles, estructuras estructuradas predispuestas para funcionar como estructuras estructurantes ${ }^{2}$, es decir, como principios generadores y organizadores de prácticas y representaciones que pueden estar objetivamente adaptadas a su fin sin suponer la búsqueda consciente de fines y el dominio expreso de las operaciones necesarias para alcanzarlos, objetivamente "reguladas" y "regulares" sin ser el producto de la obediencia a reglas (Bourdieu, 1991, p. 92).

Este concepto permite relacionar tanto lo individual como lo social; consiste también en entender cómo se enfrenta y se percibe el mundo antes de la acción, incluso corporalmente, asimismo, han inteligibles los principios generadores y organizadores, y los pre-supuestos que dan orden y norman, pero sin necesidad de una imposición de reglas o leyes en el campo jurídico. A propósito, el campo Bourdieu lo define como una red de relaciones objetivas, que son microcosmos sociales relativamente autónomos que conforman la totalidad de lo social. El campo son espacios de relaciones objetivas y contiene una configuración de estas relaciones objetivas entre posiciones, en las cuales se estructura una jerarquía de colocaciones que son ocupadas por los agentes (individuos o instituciones); distribución de posiciones que constituyen la especificidad de cada campo.

A diferencia del campo, el habitus es una estructura incorporada; es una determinada construcción históricosocial de formas y lógicas de percibir, pensar, entender y desenvolverse en cada campo.

Para Bourdieu, la violencia simbólica tiene dos características: primero, es producto de relaciones de fuerza, y segundo, impone una arbitrariedad cultural. Si bien es producto de relaciones de fuerza, no se expresa ni se muestra como una imposición, por ende, es más bien simbólica, actúa en el mundo de las re-presentaciones y se ejerce -como dice Bourdieu - en "una relación de comunicación" (Bourdieu, 1996, p. 46). Mientras que la arbitrariedad cultural es el constructo histórico-social que se naturaliza y que se impone como normal y natural, que justamente se llega a expresar en cuanto se lo aplica a la cuestión de la violencia de género, como la dominación masculina institucionalizada. A partir de lo señalado antes, esta dominación se puede entender, justamente, como las construcciones simbólicas que legitiman las construcciones histórico-sociales que son producto de relaciones de fuerza incorporadas en las relaciones entre hombres y mujeres, mismas que encubren, invisibilizan, simbólicamente, las relaciones de fuerza de la que son producto, las que benefician al hombre en desmedro de la mujer, instalando dichas relaciones como relaciones naturales.

"La fuerza especial de la sociodicea masculina procede de que acumula dos operaciones: legitima una relación de dominación inscribiéndola en una naturaleza biológica que es en sí misma una construcción social naturalizada" (Bourdieu, 2000, p. 37).

En virtud de ello, la dominación masculina se enmarca en la división social del trabajo, que se delimita en función a esa "sociodicea masculina", a esa visión del mundo y construcción institucional basada en el hombre, delimitando lugares privilegiados para este y excluyendo de estos a la mujer, como si ello fuese un hecho natural. Bourdieu, con base en su estudio de la sociedad cabilia, muestra cómo se

2 La noción de "estructuras estructurantes" es una elementos que Bourdieu utiliza frecuentemente para evitar caer en un estructuralismo secante y rígido y mostrar el papel activo de las estructuras, por eso no se replican sino que generan prácticas innovadoras. 
excluye a las mujeres de las tareas más "nobles" (manejar el arado) y se les asigna tareas inferiores, como por ejemplo actividades al margen de la carretera o terraplén, trasportar estiércol, recoger aceitunas, aprovechándose de las diferencias biológicas para ponerlas de justificativo de sus construcciones sociales (división sexual del trabajo). Esta visión del mundo se expresa en la percepción misma de las mujeres; un mundo donde se les asigna ciertas tareas y se les excluye de otras. El hombre hace uso de su posición superior de dominación naturalizada, transgrediendo sin consciencia plena a la "normalidad" (por el habitus), pues él mismo ha internalizado su posición superior, así como la mujer su posición inferior, ya que inmediatamente al nacer ambos son sumergidos en el habitus ya establecido, el cual no depende de su voluntad. Por ello, el tema de la dominación simbólica es importante, este no depende del ejercicio consciente de un poder, como es el caso de la violencia física y psicológica.

\section{Metodología}

Como parte de la metodología, se tiene el siguiente objetivo investigativo: identificar las formas y expresiones de la violencia simbólica en las experiencias de las docentes que ocuparon y ocupan cargos jerárquicos en la UMSA. Para alcanzar este objetivo, se apeló a una metodología cuantitativa-cualitativa, la cual es mixta, y que, según Denise Arnold (2010), es una buena combinación de ambas para el análisis social. Hasta el momento, se ha podido comprobar la importancia de esta perspectiva metodológica y sus importantes resultados a la hora de la investigación social (Alarcón, 2016).

Ahora bien, la metodología cuantitativa se tomó en cuenta para mostrar las diferencias de género en la presencia y conformación de docentes en las 13 facultades con las que cuenta la UMSA, y se tomó solo como ejemplo dos unidades de postgrados. En este sentido, se ha ido conformando la correspondiente unidad de análisis en términos cuantitativos.

Los datos cuantitativos fueron elaborados en colaboración y asesoría del Departamento de Sistemas de Información Estadística (DSIE) de la UMSA. Como se ve más adelante, estos datos son muy importantes, dado que presentan, en lo general, una visión panorámica para el análisis.

Para lo cualitativo, se aplicó entrevistas semiestructuradas en profundidad, pues dado que esta técnica tiene la capacidad de apertura para el diálogo; ha sido construida para dialogar sobre las experiencias personales de las entrevistadas. De ese modo, se ha entrevistado a 13 docentes mujeres, una por cada Facultad que tiene la UMSA, con base en los siguientes criterios de selección:

- Que ocuparon y ocupan cargos jerárquicos.

- Una entrevista por cada Facultad.

- Predisposición a tratar el tema.

\section{Resultados y discusión}

3.1 Reproducción de las diferencias de género en la conformación docente de la UMSA

Existe una notable diferencia entre docentes mujeres y hombres en la UMSA, tanto así que en sus 13 facultades los hombres son mayoría. A continuación, se presentan dos tablas de datos de la población docente denotando la diferencia numérica entre hombres y mujeres, además de sus respectivas categorías en todas las facultades.

Tabla 1. Conformación de docentes de las 13 facultades de la UMSA

\begin{tabular}{|l|c|c|c|c|}
\hline \multicolumn{2}{|c|}{ Porcentaje de docentes por facultad según sexo (2017) } \\
\hline \multicolumn{1}{|c|}{ Facultades } & Hombres & $\%$ & Mujeres & $\%$ \\
\hline Facultad de Medicina & 283 & 62 & 172 & 38 \\
\hline Facultad de Odontología & 72 & 72 & 28 & 28 \\
\hline Facultad de Farmacia y Bioquímica & 49 & 51 & 47 & 49 \\
\hline Facultad de Ciencias Puras y Naturales & 165 & 76 & 53 & 24 \\
\hline Facultad de Ingeniería & 486 & 91 & 49 & 9 \\
\hline Facultad de Agronomía & 146 & 81 & 34 & 19 \\
\hline Facultad de Ciencias Geológicas & 69 & 93 & 5 & 7 \\
\hline
\end{tabular}




\begin{tabular}{|c|c|c|c|c|}
\hline Facultad de Arquitectura y Artes & 141 & 75 & 47 & 25 \\
\hline Facultad Tecnológica & 379 & 91 & 37 & 9 \\
\hline Facultad de Ciencias Sociales & 274 & 62 & 165 & 38 \\
\hline Facultad de Derecho y Ciencias Políticas & 265 & 86 & 44 & 14 \\
\hline Facultad de Humanidades & 272 & 55 & 225 & 45 \\
\hline Facultad de Ciencias Económicas y Financieras & 374 & 84 & 71 & 16 \\
\hline Dirección y Coordinación de Postgrado - Cides & 15 & 60 & 10 & 40 \\
\hline Centro Psicopedagógico y de Investigación en Educación & 2 & 67 & 1 & 33 \\
\hline Totales & 2.992 & 75 & 988 & 25 \\
\hline
\end{tabular}

Fuente: elaboración propia con base a los datos estadísticos del DSIE de la UMSA.

El $25 \%$ son docentes mujeres, mientras que el restante $75 \%$ son hombres, evidenciándose la prevalencia de estos. Existen facultades donde la diferencia es notablemente mayor, llegando aproximadamente a $90 \%$ de docentes hombres en facultades como las de Ingeniería, Ciencias Geológicas y Tecnología. Pareciera que estas diferencias, entre docentes hombres y mujeres, pudieran reflejar una segmentación del trabajo por género.
De tal forma que podría pensarse en el reflejo de asimetrías, tanto en el acceso como en la permanencia de las profesionales docentes en la universidad. Los datos son claros al demostrar justamente este tipo de diferencias de género. En la siguiente tabla, puede verse la conformación relacional de docentes.

Tabla 2. Conformación de docentes de las 13 facultades en relación a sus categorías

\begin{tabular}{|c|c|c|c|c|c|c|c|c|c|}
\hline \multirow[b]{2}{*}{ Categoría } & \multicolumn{3}{|c|}{ Octubre 2016} & \multicolumn{3}{|c|}{ Octubre 2017} & \multicolumn{2}{|c|}{ Marzo 2018} & \multirow[b]{2}{*}{ Total } \\
\hline & Masculino & Femenino & Total & Masculino & Femenino & Total & Masculino & Femenino & \\
\hline Contratado & 126 & 41 & 167 & 123 & 33 & 156 & 130 & 42 & 172 \\
\hline Interino & 606 & 235 & 841 & 705 & 285 & 990 & 531 & 264 & 795 \\
\hline Invitado & 513 & 197 & 710 & 489 & 213 & 702 & 521 & 156 & 677 \\
\hline Titular A & 291 & 76 & 367 & 272 & 77 & 349 & 269 & 77 & 346 \\
\hline Titular B & 484 & 143 & 627 & 513 & 157 & 670 & 522 & 163 & 685 \\
\hline Titular C & 297 & 88 & 385 & 248 & 81 & 329 & 257 & 79 & 336 \\
\hline T. Emérito & 684 & 192 & 876 & 757 & 207 & 964 & 745 & 204 & 949 \\
\hline Total & 3.001 & 972 & 3.973 & 3.107 & 1.053 & 4.160 & 2.975 & 985 & 3.960 \\
\hline
\end{tabular}

Fuente: elaboración propia con base a los datos estadísticos del DSIE de la UMSA.

Con estos datos, se puede comprobar que la tendencia a lo largo de los años no varía significativamente, sin embargo, se evidencia la diferencia de género en la conformación docente en las 13 facultades, tanto a nivel de presencia como en relación con las categorías docentes, lo que permite percibir un contexto marcadamente masculino al que la que una mujer se debe enfrentar a la hora de ejercer algún cargo jerárquico.
Como se aprecia, en las gestiones de los años 2016, 2017 y 2018 la presencia masculina, en términos totales, ha sido de alrededor de 3.000 docentes, mientras que la presencia de docentes femeninas está en torno a las 1.000. Esto significa que, en comparación, la presencia masculina triplica la femenina en la universidad. Hay una docente por cada 3 docentes hombres. En cuanto a las categorías docentes, como puede verse en la de Interino, la mayoría de docentes 
son hombres, lo propio sucede en la categoría Titulares, donde se ven grandes diferencias y existe una abrumadora mayoría de docentes hombres.

\subsection{Percepciones de discriminación como expresión de la violencia simbólica}

A continuación, se mostrarán las percepciones de discriminación que las entrevistadas manifestaron en relación con sus experiencias vividas en el ejercicio de sus cargos jerárquicos. Luego, se verá la forma cómo estas percepciones expresan el tipo de violencia simbólica; y de ese modo mostrar, posteriormente, cómo las entrevistadas responden a esta situación de manera no consciente, es decir, las actitudes que optan como defensa sin tener consciencia de la forma de su propia actitud ante estas situaciones de violencia.

\subsection{Actitudes de menosprecio}

Estas actitudes son relatadas como acciones que desvalorizan la participación de las mujeres en el ejercicio de cargos jerárquicos, es decir, al fungir como autoridades en la UMSA. Veamos algunas expresiones de las docentes entrevistadas.

Cuando fui vicedecana, en las reuniones de trabajo se experimentaron las imposiciones de los varones en la toma de decisiones. Ellos siempre son los que tienen la razón y la experiencia (...) No dejan participar (...) Ellos tenían una actitud bastante relajada, una posición de cuerpo relajada, se colocaban sus lentes oscuros; y seguramente querían cerrar sus ojos y dormir. (Entrevista 43)

Estas actitudes percibidas por la entrevistada demuestran, en principio, como una afrenta a las capacidades de las mujeres, donde el cargo (vicedecana, en este caso) no frena este tipo de actitudes contra la mujer. En todo caso, puede advertirse el menosprecio; como que a las autoridades mujeres no se las debe escuchar, dado que al parecer lo que tienen que decir carece de valor. Si bien se naturalizan este tipo de actitudes, responden en el fondo a un tipo de estructura que tiene sus defensores.
Estas no son acciones directas, debido a que, como la mujer, con su presencia, interrumpe la normalidad de la ocupación de los espacios de toma de decisión esta irrupción es sancionada sutilmente; y como el hombre sigue siendo mayoría en dichos espacios que le otorgan poder, seguirá reproduciendo las indicadas actitudes. Todo pasa como si la mujer fuera la del problema, misma que no tiene más que enfrentar situaciones adversas en sus fuentes laborales en general y particulares cuando fueron y son autoridades. A continuación, se presenta un relato.

En el cargo de vicedecana de Ciencias Puras y naturales, mis opiniones como mujer joven en el cargo no se tomaban como opiniones serias. Me ha tomado mucho tiempo en hacer conocer mi capacidad, toda la experiencia; en demostrar que lo que yo opinaba era cierto y útil (...) No lo dicen, pero uno lo percibe. (Entrevista 3)

Estas experiencias, de alguna forma han sido expresadas por otras entrevistadas. La figura es la misma, son acciones que por no ser directas solo se perciben sin embargo cumplen una función concreta, que consiste en hacer sentir a la mujer que su presencia dentro de cargos que normalmente son ocupados por hombres no son para ellas; y no son aceptas. Otra entrevistada relató lo siguiente:

En el 93 era candidata a decana. Voto mayoritario estudiantil, teníamos; pero perdimos por los votos docentes (...) Noté el rechazo por el género y por generación. Recién me di cuenta del efecto de la campaña sucia que me hicieron los docentes, especialmente los más mayores. (Entrevista 13)

De ese modo, se va dando forma a una serie de perspectivas introducidas en la sociedad universitaria. Son, en este caso, las mismas mujeres docentes las que mantienen un lenguaje para percibir cuando la violencia simbólica opera sobre ellas. Por ejemplo, en frases como "no nos toman en serio", "ocultan el aporte de la mujer", "es más fácil censurar a una mujer", "ese es un pensamiento femenino; y con ese pensamiento no vamos a avanzar", "ella no sabe nada, no sabe dónde está parada"; y así, siguen.

3 Por un tema de ética, en las citas extraídas de las entrevistas realizadas se respetó el lenguaje coloquial de las profesoras entrevistadas. 


\subsection{Incapacidad "impuesta"}

Este aspecto tiene que ver con las acciones concretas que los hombres realizaron contra las mujeres, obstruyendo y dificultando el desenvolvimiento de sus actividades laborales, construyendo así una imagen de "incapacidad" de la mujer para ejercer cargos jerárquicos.

El desprecio a la mujer es mucha. De hecho, ahora tenemos, excepcionalmente una vicedecana en la Facultad de Derecho, que es autoridad y abogada y al mismo tiempo docente de la carrera de Ciencias Políticas (...) Creo que es la primera vez en la historia de la Facultad de Derecho que hay una autoridad mujer y abogada. Entonces, por ejemplo (...) ella es la única mujer del Consejo Facultativo; y uno ve permanentemente cómo (...) los colegas que son jerárquicamente inferiores (...) la menosprecian. (Entrevista1)

Estas son acciones concretas que afectan el ejercicio laboral de las mujeres en sus cargos; $y$ lo que se percibe es que los móviles de dichas acciones son machistas.

Este trabajo no es para mujer. Yo era la única mujer cuando estaba en Patacamaya; ${ }^{4}$ y por eso me asignaron la parte de laboratorio. Hacía una cosa de siembra, hacía mi jardín. Entonces los técnicos a propósito le echaban más cantidad de fertilizante y me dañaban todas mis plantas. (Entrevista 7)

Estas acciones rebasan las jerarquías; es como si la relación hombres/cargos jerárquicos fuera natural, y cuando se interrumpe dicha relación el nivel jerárquico de cargos se suspende. De ese modo nos cuenta una entrevistada sobre la relación laboral con su colega.

Había un jefe que me hostiga laboralmente. Él quería que yo fuera a trabajar a las seis y media de la mañana, a las siete y media de la mañana. Reclamaba de algunas notas que no se habían enviado; y entonces yo llevaba el libro y decía: "Ya se había vuelto a reenviar la nota con la carta de respuesta"... Un jefe que era por debajo de mí, que dependía de mí... A raíz de eso, yo le hice una nota de reclamo contra su acoso laboral, porque no era quien para que me dijera que yo debía estar ocho menos cuarto o mucho más antes, porque la entrada es a las nueve, tomando atribuciones que no le correspondían... Las mujeres, a veces, cuando asumimos esos cargos, apoyadas por una dirección, piensan ellos, los hombres, que somos marionetas. (Entrevista 4)

\subsection{Violencia sexual implícita}

Se pudo ver que, pese al cargo ocupado, la percepción sobre la mujer como un objeto del deseo persiste; y en muchos casos de una manera poco acorde con los protocolos institucionales, por ejemplo:

Yo no solo he presenciado, sino he sufrido violencia intimidatoria de orden sexual, (...) hay muchos colegas que cuando una está en una condición de decisión o de representación en algún lugar jerárquico, empiezan a hacer una especie de coqueteo a la mujer, para mimetizar, entre comillas, la violencia; y surgen insinuaciones... La situación de soltería, en una mujer de la universidad, es malinterpretada, da paso a que muchos varones, fueran de la condición que fueran, hagan a la mujer insinuaciones... La situación de no pertenencia a un varón, da paso a estas insinuaciones. (Entrevista 1)

Estas acciones son percibidas por las mujeres como un tipo de violencia hacia ellas, como se vio en relato anterior. Las entrevistadas perciben que se vulnera y pone en duda su capacidad, ya que, haciendo alusión a cuestiones físicas y estéticas, el papel que juegan en el ejercicio de cargos jerárquicos aparece minimizado, como se refleja en el siguiente comentario de una ex-vicedecana:

... por ejemplo, de pronto en una reunión ejecutiva uno no se puede poner la falda ${ }^{5}$ porque le miran las piernas, o te dicen "habías tenido buenas piernas", como alguien me lo dijo. Cuando yo subí a hablar a la testera, al bajar, me dijeron "que buenas piernas habías tenido", una cosa así; y venía de una persona con bastante jerarquía. (Entrevista 4)

Estas acciones, si bien pueden ser tomadas como menosprecio (en este caso no tomando en cuenta la

4 Pequeña ciudad y municipio de Bolivia perteneciente al departamento de La Paz.

5 Falda en Bolivia se refiere a un vestido, enagua o pollera. 
exposición de la vicedecana y, más bien, resaltando cuestiones estéticas), muestran también el papel de "conquistador" que socialmente se predispone para el hombre en su condición de agente activo, en relación con la mujer como agente pasivo en las relaciones amorosas, pero que, dentro de las relaciones laborales, toma otro tipo de connotaciones; y las mismas mujeres lo perciben como humillantes.

Algo que creo todas las mujeres hemos enfrentado, lamentablemente, en el ejercicio de la profesión, que es común de los hombres hacia mujeres, es el piropo. Es este piropo que, en vez de ser halagador, es más bien ofensivo y está entendido también como violencia. En el ámbito laboral siempre se vive muchas veces esta situación, ¿no? Con abogados, con policías siempre había como una, como una mención a una cuestión personal. (Entrevista 8)

En ocasiones... se me criticaba el ir con falda a la facultad y era sujeto de muchos piropos que eran, así, insultantes para mi persona, por lo cual creo que mi reacción ha sido de mucha molestia... Sí, veo que lo hacen todavía con docentes que son jóvenes y molestan bastante en su integridad, sobre todo. (Entrevista 10)

Estas acciones pueden solamente ser tomadas como de "coquetería", de "seducción", pero que llevadas al ámbito laboral se perciben como machistas, desagradables y ofensivas. Al llevar la relación de la "conquista" amorosa, donde el hombre asume el papel activo y la mujer el pasivo, ${ }^{6}$ pareciera que las relaciones laborales se transfiguran con ese otro ámbito. Así, la mujer percibe un intento de dominación, de violencia por medio de esos actos de seducción, lo que también ocasiona que el nivel jerárquico entre en suspensión, como si otro orden social instituido pesara más y buscara normalizarse sin importar ámbitos -el laboral, en este caso-, papeles ni jerarquías para reproducirse otra vez.

\subsection{Locas y brujas}

Hay algunos ejemplos que muestran el uso de los términos locos y brujos con intenciones de descalificar las actitudes de las mujeres; y más aún de sancionar la interpelación que ellas hacen, con su presencia, al entorno machista, ocupando cargos que se consideran solo para hombres.

Ha habido un desprecio hacia algunas mujeres, algunas docentes, cuando se encontraban en reuniones de la asociación. Cuando una mujer tomaba la palabra, siempre un hombre decía "ella no sabe nada, no sabe dónde está parada..."; o el decirnos locas o brujas. (Entrevista 4)

Como existe una normalidad instituida, un "orden de cosas", una estructura que pone a los hombres en cargos directivos jerárquicos, la transgresión a este orden provoca reacciones masculinas. Otro ejemplo es el siguiente:

Hemos ganado un concurso a un ingeniero, y por eso le estábamos asesorando a un estudiante; y este estudiante le había comentado que quería hacer su proyecto sobre algo de medio ambiente. $Y$ ahí vienen las expresiones de esos docentes: "iAh!, no me vegas con ese tema. Estás hablando como esas dos locas". Entonces, yo era una loca; la otra loca era la coordinadora del proyecto. $Y$ lo dijo delante de todos los docentes. (Entrevista 6)

Este término -locas - puede pasar como un adjetivo normalizado, sin evidenciar directamente su carácter machista, pero en el fondo es una expresión de violencia simbólica, pues se va haciendo como un apelativo último que denota claramente discriminación.

En este relato se ve que el término estaba ligado a menospreciar las capacidades de las académicas, mostrando que lo que ellas hacen y dicen no solo no tiene importancia, sino que son cosas sin sentido, cosas de personas locas, lo que busca reafirmar el orden masculino de las cosas, y a partir de ello menospreciar las capacidades de las mujeres.

\subsection{Los chistes machistas}

Si bien no se narraron ninguno de estos chistes, algunas entrevistadas contaron que es así como se siente el machismo, mediante los chistes.

6 "Si la relación sexual aparece como una relación social de dominaciones porque se constituye a través del principio de división fundamental entre lo masculino, activo, y lo femenino, pasivo, y ese principio crea, organiza, expresa y dirige el deseo, el deseo masculino como deseo de posesión, como dominación erótica, y el deseo femenino como deseo de la dominación masculina" (Bourdieu, 2000, p. 35). 
He notado que esas actitudes se traducen mediante el chiste (...) Se denotan las actitudes machistas con chistes. Esto ocasiona que muchas colegas se inhiban de participar, porque no es agradable. (Entrevista 2)

Hay que tener claro que, tanto hombres como mujeres, nacemos bajo ciertas condiciones, construcciones históricosociales ya instauradas, mismas que nos determinan. Este mundo instituido, que para Bourdieu son "condiciones objetivas", relaciones de fuerzas institucionalizadas, que se conforman en función de la relación entre dominantes y dominados. La violencia simbólica legitima esas relaciones de fuerza; transfigura las condiciones histórico-sociales objetivas, que son la imposición de unos sobre otros. Y no solo las pone como "el orden de las cosas", sino que ayuda a perpetuarlas; no de manera directa, violenta, sino simbólica o implícitamente, sancionando así la transgresión a ese orden.

Como ya se vio, el habitus determina a los agentes (hombres/mujeres) más allá de la consciencia y de su voluntad. Podemos ser conscientes de la discriminación contra la mujer y tener la voluntad de cambiarla, pero aun así se continúa reproduciendo, por eso esto sigue siendo un problema. Según las percepciones de las entrevistadas, los hombres sienten vulnerada su posición dominante cuando con su presencia una mujer ocupa un cargo que "normalmente" es de hombres. He aquí un ejemplo:

Ven a la mujer como amenaza para su certidumbre de privilegios; y es algo muy importante. Los hombres en general, en los espacios laborales en Bolivia, tienen un privilegio mayor (...) por su simple condición de ser hombres. Cuando una mujer está de igual a igual en un espacio laboral, se sienten intimidados por la presencia de la mujer. $Y$, ¿a qué se refiere esta intimidación? A que pierden espacios de poder. (Entrevista 1)

Esta vulneración a la "normalidad" del entorno machista, que el hombre no deja pasar, no es consciente. Es difícil pensar no solo que el hombre sea plenamente consciente de que la mujer esté transgrediendo el "orden de las cosas" que lo privilegia, sino, peor aún, que sea capaz de responder a dicha transgresión elaborando un chiste que sirva como sanción para hacer sentir a la mujer la transgresión al entorno machista que ella hace con su presencia en espacios "reservados" socialmente para el hombre.

Dentro de las relaciones de género, somos parte de construcciones histórico-sociales de corte machista. Cuando el goce de cierto poder o beneficios es vulnerado, es evidente que el beneficiario de estos reaccione; y dicha reacción, casi automática, se puede expresar en la forma de chiste.

\subsection{Estrategias no conscientes frente a la violencia simbólica}

El habitus predispone la práctica social, pero dentro de esta son los agentes (hombres o mujeres) quienes asimilan dicha predisposición en función del acontecimiento. ${ }^{7}$ En tal sentido, el habitus solo es la condición de posibilidad de las prácticas. Esta imprevisibilidad (producto del acontecimiento) permite explicar ciertas reacciones de las mujeres, como estrategias no-conscientes, que se expresan en la forma en que las mujeres asimilan el entorno machista, en otras palabras, son las formas como las entrevistadas han respondido a todo el entramado de percepciones negativas que se tienen en contra de ellas. A continuación, se mostrarán los elementos de esta estrategia no consciente, reflejada en las entrevistas, para después explicar cómo se relacionan.

\subsection{Autoafirmación}

Se llama autoafirmación a las opiniones emitidas por las entrevistadas que indicaban que las mujeres cuentan con mejores cualidades para desempeñar cargos jerárquicos.

No es lo mismo. El hombre tiene un liderazgo en lo que significa ser autocrático; la mujer es mucho más democrática, sabe escuchar; es mucho más participativa (...), es más accesible, confía más, quizá por el tema de ser madre; la constitución ${ }^{8}$ que nos da de ser madre, entendemos más a la gente. En cambio, al hombre le resbalan muchos sentimientos. (Entrevista 4)

7 "Si la génesis del sistema de las obras o las prácticas engendradas por el habitus (o por habitus homólogos como los que constituyen la unidad del estilo de vida de un grupo o de una clase) no puede ser descrita como desarrollo de una esencia única y siempre idéntica a sí misma" (Bourdieu, 1991, p. 96-97).

8 En este caso hace alusión a la condición natural de ser madre. 
Si bien los hombres pueden además autoafirmarse, indicando que cuentan con mejores capacidades, en las entrevistas esta autoafirmación se hace en relación con el género, es decir, con el hecho de ser hombre. No se la efectúa como un tema individual, donde se busque mostrar la valía personal en el ejercicio de un cargo, sino como una cuestión de género en general.

La mujer es más detallista; las cosas las hace con más detalle y las hace más pulcras. Mientras que el varón tiene flojera de revisar algunas cosas y deja pasar. Su opinión es diferente de "ella". Nosotras cuidamos esas cosas en detalle, incluso para poder opinar. (Entrevista 7)

Es necesario resaltar que se apela, en muchos casos, a la condición de ser madres, así se refleja una autoafirmación de género biológicamente fundamentada. Es preciso mencionar que se encontró un caso donde se expresó que las diferencias de sexo no determinan el ejercicio de ninguna actividad, como se ve a continuación:

Ya estamos en una etapa muy avanzada: la igualdad de género. Se dice que todos somos iguales, pero yo pongo mis límites. Cuando quiero mover un mueble, cuánta falta me hace la fuerza de un varón. Pero, ya después, para el ejercicio de cualquier actividad, actividades de toda naturaleza, no hay diferencia entre hombre y mujer. (Entrevista 5)

\subsection{Doble responsabilidad}

Encontramos que otras de las estrategias consiste en asumir el cargo de manera óptima y mostrar que son mejores que los hombres. Se trata también de que las mujeres no solo tienen que ejercer el cargo bien, sino que deben ejercerlo mejor que los hombres. Pareciera que esta conclusión —palabras más palabras menos- se puede leer entre líneas en casi todas las entrevistas. La mujer asume una responsabilidad doble cuando acepta un cargo jerárquico, pues no solo debe estar a la altura de dicho cargo, sino que debe desempeñarlo pensando en su condición de mujer, ejerciéndolo con mayores niveles de presión impuesta por ellas mismas, como lo expresa a continuación otra entrevistada.

Yo le dije: "Aquí hay discriminación". Uno tiene que demostrarse superior a ellos, para que te consideren igual. (Entrevista 9)
La doble responsabilidad que se adjudican las entrevistadas es una cuestión más allá de lo individual; involucra el género, pues ellas mismas asumen el cargo con la responsabilidad extra de ser un ejemplo para otras mujeres.

Es bueno, por la visión que se tiene desde afuera; otras mujeres siguen el ejemplo. Una motiva a otras. (Entrevista 3)

El papel de género que asumen es claro; buscan reivindicar las condiciones negativas que ellas enfrentan.

No es indiferente que en otros espacios de investigación (...) existan mujeres que lideren los procesos de investigación. Para mí es absolutamente relevante no solamente para que se reconozca, sino que para que no se oculte. Lo que normalmente pasa en la práctica de la investigación científica en general (...) es que no se reconoce, siempre se oculta. Hay un ocultamiento —vamos a decir así- ideológico premeditado. La mayor parte de los premios Nobel son hombres, a pesar de que sí existían mujeres que se los merecían. (Entrevista 1).

Pareciera que este fuese un deber "encarnado", que ante las estructuras, prácticas y percepciones machistas sea necesario realizar una forma de lidiar con la discriminación.

\section{Conclusiones}

En cuanto a la diferencia de género, la UMSA es, en términos cuantitativos, desigual, o sea, que hay una presencia abrumadora de hombres docentes. Lo mismo se da en las categorías (diferencia contractual, ya sea como contratado, interino o titular). De ahí que las entrevistadas perciban todo tipo de sensaciones adversas, justificadas, pues como se mostró existen varias experiencias que indican una especie de dominación masculina hacia las mujeres.

El menosprecio, la incapacidad impuesta, la violencia simbólica están implícitos; sus formas y expresiones son evidentemente demostradas en las experiencias de las docentes cuando remarcan temas analizados, como el de los chistes y el calificativo de "locas", que dentro del campo de la educación superior son construcciones históricosociales producto de relaciones de fuerza, que en este caso favorecen a la dominación masculina o, más bien, son predisposiciones que no se manifiestan en acciones 
directas, sino que son sutiles y están presentes como parte de la cultura local.

Las entrevistadas perciben que no se puede probar que las opiniones emitidas por una mujer en un espacio de toma de decisiones se tomen a menos y desvaloricen solo por el hecho de que es ella la que habla. Al no poder lidiar directamente con las experiencias negativas que narraron, pues son formas sutiles de dominación, formas simbólicas, se enfrentan a ellas de la mejor manera que pueden, pero individualmente, de ahí que se carguen el peso de realizar un trabajo doble o deresponsabilidad doble.

Las susceptibilidades siempre están presentes y se confirman con las acciones concretas de menosprecio, incapacidad construida y la violencia implícita. Esto se confirma con el habitus, pues más allá de que las mujeres busquen un empoderamiento y sean conscientes de las condiciones adversas impuestas por los hombres y demás obstáculos de un entorno machista se nace (tanto hombres como mujeres) con condiciones que determinan las prácticas y percepciones sociales, lo que está fuera de su consciencia y voluntad.

Desde el análisis que Sigmund Freud hace sobre el chiste, encuentra una similar construcción de este en relación con la elaboración del sueño. Básicamente éste produce un placer, que es causado por la satisfacción de no reprimir algo o de saltar un obstáculo que causa una represión; ese algo del que se habla puede ser un instinto hostil. ${ }^{9}$ El uso del chiste o la broma también evitaría la sanción social que puede aplicarse contra un hombre si este emitiera o manifestara un juicio o afrenta verbal sin la mediación y transfiguración que pueda hacer está. El punto aquí es pertinente para explicar los chistes con tendencia machista, pero el tema del chiste y el análisis que hace Freud se enfocan en relación con la elaboración del sueño; y es lo que permite entender por qué el chiste puede ser considerado como una manifestación de violencia simbólica, pues esta no solo se explica por el placer que produce, sino porque su aparición es el resultado de un proceso que transforma un entramado ideológico, para ser expresado de manera que oculta dicho entramado; y que según Freud esto no se lo hace conscientemente, pues un pensamiento pre-consciente (que podría relacionarse con el habitus) pasa al inconsciente donde se transfigura para mostrarse a la percepción consciente. ${ }^{10}$

Este aspecto resaltado es un juego simbólico, pues el chiste representa ideas que no son expresadas directamente; y no solo eso, sino que se lo hace de manera no consciente, punto que se muestra también con el concepto de habitus, gobernando al sujeto más allá de la voluntad y la consciencia. Es posible entender de mejor manera el carácter simbólico del chiste mediante el análisis de Freud. A pesar de ello, es necesario mencionar el carácter hipotético de esta consideración y sugerir una investigación más a fondo sobre este tema específico.

\section{Referencias}

Alarcón, Valentina Rosario (2016). "La formación de líderes políticos nacionales, conciencia histórica y aspectos psicosociales". Tesis doctoral. La Paz-Bolivia: CidesUMSA.

Arnold, Denisse; Spedding, Alison; Pereira, Rodney (2006). "Pautas metodológicas para investigaciones cualitativas y cuantitativas en Ciencias Sociales y Humanas". La Paz-Bolivia: U-PIEB.

Bourdieu, Pierre (1991) El sentido práctico. Madrid-España: Editorial Taurus.

(2000). La dominación masculina. Barcelona-España: Editorial Anagrama.

Bourdieu, Pierre y Passeron, Jean Claude (1996). La reproducción: elementos para una teoría del sistema de la enseñanza. México: Ed. Fontamara,

Bourdieu, Pierre y Wacquant, Loïc (1995). Respuestas: por una antropología reflexiva, Ed. Grijalbo, México.

Freud, Sigmund (1970) El chiste y su relación con lo inconsciente. Madrid-España: Alianza Editorial.

9 "El chiste representa una rebelión contra tal autoridad, una liberación del yugo de la misma... el chiste puede atacar igualmente aquellas instituciones, personas representativas de las mismas, preceptos morales o religiosos o ideas" (Freud, 1970, p. 91).

10 "La regresión del proceso mental a la percepción falta seguramente en el chiste; más los otros dos estadios de la elaboración de los sueños, el descenso de un pensamiento pre-consciente a lo inconsciente y la elaboración inconsciente, nos proporcionarían, transportados a la elaboración del chiste" (Freud, 1970, p. 148). 